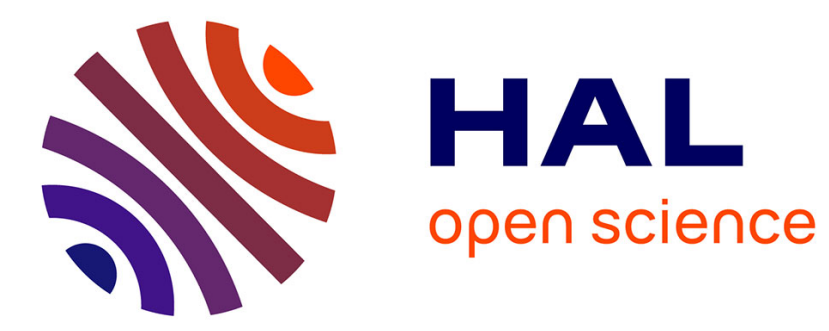

\title{
Human promoter mutations unveil Oct-1 and GATA-1 opposite action on Gfilb regulation
}

\author{
Aurora Hernández, Ana Villegas, Eduardo Anguita
}

\section{To cite this version:}

Aurora Hernández, Ana Villegas, Eduardo Anguita. Human promoter mutations unveil Oct-1 and GATA-1 opposite action on Gfilb regulation. Annals of Hematology, 2010, 89 (8), pp.759-765. 10.1007/s00277-009-0900-x . hal-00535122

\section{HAL Id: hal-00535122 \\ https://hal.science/hal-00535122}

Submitted on 11 Nov 2010

HAL is a multi-disciplinary open access archive for the deposit and dissemination of scientific research documents, whether they are published or not. The documents may come from teaching and research institutions in France or abroad, or from public or private research centers.
L'archive ouverte pluridisciplinaire HAL, est destinée au dépôt et à la diffusion de documents scientifiques de niveau recherche, publiés ou non, émanant des établissements d'enseignement et de recherche français ou étrangers, des laboratoires publics ou privés. 


\title{
Human promoter mutations unveil Oct-1 and GATA-1 opposite action on Gfilb regulation
}

\author{
Aurora Hernández • Ana Villegas • Eduardo Anguita
}

Received: 3 August 2009 /Accepted: 30 December 2009/Published online: 9 February 2010

(C) Springer-Verlag 2010

\begin{abstract}
Growth factor-independence $1 \mathrm{~b}$ (Gfilb) is a zinc finger transcription factor essential for erythroid and megakaryocytic development. To better understand Gfilb regulation and to know the implication of the level of expression of this gene in human pathology, we have searched for promoter punctual sequence variations in 214 patients with different hematological diseases. We found two previously unknown congenital mutations at evolutionary conserved GATA and octamer-binding (Oct) transcription factor sites. The Oct site mutation was also found in five relatives of the patient. The GATA motif mutation reduced promoter activity by $50 \%$ in vitro, while homozygous patients with the octamer site mutation showed a four-to-five times increase of Gfilb RNA in platelets. Electrophoretic mobility shift analyses demonstrated that different protein complexes bind to both sites and that binding is reduced by the mutations. Finally, we found that GATA-1 and Oct-1 are the main components of each complex. This study provides evidences of a new mechanism for Gfilb repression. This is also the first report of Gfilb mutations with a functional implication; further investigation and follow-up will clarify the involvement of these mutations in hematological disease.
\end{abstract}

Keywords Gfilb · Mutation - Gene regulation · Leukemia

Electronic supplementary material The online version of this article (doi:10.1007/s00277-009-0900-x) contains supplementary material, which is available to authorized users.

A. Hernández $\cdot$ A. Villegas $\cdot$ E. Anguita $(\bowtie)$

Hematology Department, Hospital Clinico San Carlos,

University Complutense,

28040 Madrid, Spain

e-mail: eduardo.anguita@imm.ox.ac.uk

\section{Introduction}

Transcription factor growth factor-independence $1 \mathrm{~b}$ (Gfilb) is expressed in hematopoietic stem cells, common myeloid and megakaryocyte/erythroid progenitors, and erythroid and megakaryocytic lineages. Moderate levels of expression are found in immature B cells, a subset of early $\mathrm{T}$ cell precursors and peripheral blood granulocytes and monocytes $[1,2]$. During erythroid differentiation, Gfilb is upregulated in early erythroblast stages and decreases with terminal differentiation $[1,3,4]$. Knockout mice have demonstrated that this gene is required for development of both erythroid and megakaryocytic lineages [5]. Additionally, Gfilb expression has been found to increase in leukemia patients and cell lines $[6,7]$.

These facts indicate that correct Gfilb expression is important to achieve normal erythroid and megakaryocytic differentiation. Therefore, it is crucial to understand how Gfilb is regulated.

Here, we have identified two different congenital Gfilb promoter mutations. These mutations reveal the presence of different protein complexes, including GATA-1 and Oct-1, involved in Gfilb regulation, giving clues of Gfilb repression mechanism. Sequence variations like these ones together with additional abnormalities are likely to have an impact in hematological disease.

\section{Methods}

Patients

We have studied 128 cases of acute leukemia of all FAB types (107 analyzed at diagnosis and 21 of them in relapse); 69 patients with acute myeloid leukemia (AML): 7 AML-M0, 12 AML-M1, 11 AML-M2, 6 AML-M3, 13 AML-M4, 16 AML- 
M5, 2 AML-M6, 2 AML-M7, and 59 cases of acute lymphoblastic leukemia (ALL): 46 B lineage ALL and 13 cases of T-ALL. We also analyzed eight myelodisplastic syndromes; two myelofibrosis; five congenital dyserythropoietic anemia (CDA) type II; one CDA type III; one case of "variant CDA type II" with negative Ham test [8, 9]; one case of CDA with thrombocytopenia; one congenital sideroblastic anemia (the last three patients had normal GATA-1 by exon sequencing; data non shown); 36 patients with thrombocytosis (platelets between 429 and 1,437 10 $3 / \mu \mathrm{l}$ ), 15 of them with JAK 2 V617F mutation, detected as described [10], and 31 with polyglobulia, 22 males with hemoglobin $(\mathrm{Hb})$ range 17.7 to $18.6 \mathrm{~g} / \mathrm{dl}$ and 9 females with $\mathrm{Hb}$ between 15.2 and $19.4 \mathrm{~g} / \mathrm{dl}$. Only ten individuals with polyglobulia had JAK 2 V617F mutation that confirmed the diagnosis of polycythemia vera; the other cases were studied to investigate the involvement of Gfilb promoter single nucleotide polymorphisms (SNPs) in polyglobulias that cannot be classified as myeloproliferative disorders.

\section{DNA isolation}

Samples were isolated from bone marrow (BM) or peripheral blood (in the cases of thrombocytosis, polyglobulia, myelofibrosis, and 24 acute leukemia patients with $60-94 \%$ blasts in peripheral blood in which BM was not available) and DNA extracted by phenol/chloroform.

\section{Point mutation analysis}

The Gfilb promoter was amplified by polymerase chain reaction (PCR) with AmpliTaq Gold (Applied Biosystems, ABI, Foster City, CA, USA) and Pwo DNA Polymerase (Roche, Basel, Switzerland) with the following nucleotides:

\section{5'-AAAGCGTGCCGCTCCAAGTG-3' \\ 5'- ACAGTGGCTCAGACGAGGCTACGTC-3'.}

The resulting products were denatured and then reannealed to allow heteroduplex formation. Afterward, PCR fragments were analyzed by denaturing high-performance liquid chromatography (DHPLC) in a WAVE 3500HT Fragment Analysis System (Transgenomic, Omaha, NE, USA). When an abnormal WAVE pattern was detected, we performed direct sequencing with the same primers and BigDye terminator sequencing kit (ABI) in an $\mathrm{ABI}$ Prism 3100 genetic analyzer.

SNPs were confirmed in at least another PCR, cloned in pGEM-T easy vector system (Promega, Madison, WI, USA), and sequenced.

Cell culture

L8057 cells, which have some megakaryocytic characteristics, and K562 and MEL 585 erythroid cells were grown and differentiated as described $[11,12]$. Primary human erythroblasts were obtained as described [13, 14]. Briefly, peripheral blood mononuclear cells were isolated by ficoll gradient and cultured in phase I medium: minimum essential medium (MEM) alpha modification (Sigma, St Louis, MO) with $1.5 \mathrm{mmol} / 1$ glutamine, $100 \mathrm{U} / \mathrm{ml}$ penicillin, $0.1 \mathrm{mg} / \mathrm{ml}$ streptomycin (both from Gibco BRL, Rockville, MD), 10\% fetal calf serum (FCS; Biosera, East Sussex, UK), $1 \mu \mathrm{g} / \mathrm{ml}$ cyclosporine A (Novartis, Basel, Switzerland), and 10\% conditioned medium from 5,637 bladder carcinoma cells. After 7 days in culture, nonadherent cells were incubated in phase II medium $\alpha$-MEM with $30 \%$ FCS with $1 \mathrm{U} / \mathrm{ml}$ human erythropoietin (Janssen-Cilag, New Brunswick, NJ, USA), $10^{-6} \mathrm{M}$ dexamethasone (Merck, Whitehouse Station, NJ, USA), $1 \%$ deionized BSA, $10^{-5} \mathrm{M} \beta$-mercaptoethanol, $0.3 \mathrm{mg} / \mathrm{ml}$ holo-transferrin human, and $10 \mathrm{ng} / \mathrm{ml}$ human recombinant stem cell factor (last four from Sigma) for 13 days.

Promoter functional study

Alleles with promoter mutation and normal control were amplified with the following primers:

\section{5'-TGCAGATGTTATTCAAAATTTCC-3' 5'-CTGTCAACTGTGTGACTCTTTGC-3'.}

PCR products were cloned in pGL3-Enhancer vector (Promega) and sequenced.

K562 cells were transiently cotransfected in a Bio-Rad Gene Pulser Electroporator $(960 \mu \mathrm{F}, 220 \mathrm{mV})$ with $10 \mu \mathrm{g}$ of each promoter construct and $1 \mu \mathrm{g}$ of pEGFP-C2 (Clontech, Mountain View, CA) control.

Luciferase activity was determined with Dual-Luciferase Reporter Assay (Promega). Green fluorescent protein (GFP) expression was analyzed in a Dako (Glostrup, Denmark) flow cytometer.

RNA isolation and semiquantitative and quantitative real-time PCR

RNA was extracted with Tri reagent (Sigma) and transcribed with MuLV reverse transcriptase (ABI) and primer random (Roche).

The cDNA was amplified in a reaction containing Taqman Universal PCR Master Mix (ABI) and appropriate primers and FAM-NFQ probe: glycoprotein VI, Hs00212574_m1; platelet glycoprotein IIb; Hs00166246_m1; platelet glycoprotein IIIa, Hs00173978 m1; Gfilb, Hs00180261 m1 and transferrin receptor, 4333770; $\beta$-actin, 4333762, and glyceraldehyde 3-phosphate dehydrogenase (GAPDH), 4333764, endogenous controls (Gene Expression Assays, ABI). Realtime PCR expression was calculated relative to GPVI and GAPDH or $\beta$-actin using the $\Delta \mathrm{C}_{\mathrm{T}}$ method. Statistical 
Table 1 Oligonucleotides used in EMSA studies $(\mathrm{WT}=$ wild type)

\author{
Gfilb promoter WT \\ Sense \\ Antisense \\ Mutant -8099 (A-G) \\ Sense \\ Antisense \\ Mutant -8092 (T-C) \\ Sense \\ Antisense
}

5'-GGAAAGTTTTGATAAGCAAATACGGCTGAGCT-3' 5'-GGAGCTCAGCCGTATTTGCTTATCAAAACTTT-3'

5'-GGAAAGTTTTGATGAGCAAATACGGCTGAGCT-3' 5'-GGAGCTCAGCCGTATTTGCTCATCAAAACTTT-3'

5'-GGAAAGTTTTGATAAGCAAACACGGCTGAGCT-3' 5'-GGAGCTCAGCCGTGTTTGCTTATCAAAACTTT-3' analysis was performed with nonparametric Mann-Whitney test and SPSS 15.0 program. A $P$ value $<0.05$ was considered significant.

Electrophoretic mobility shift analyses

DNA binding proteins were extracted from $10^{7}$ induced MEL and L8057 cells as described [15]. Electrophoretic mobility shift analysis (EMSA) was performed as described [16]. Consensus GATA, Oct, and Gfilb binding control oligonucleotides have been previously published [17-19]. The other oligonucleotides used are shown in Table 1.

\section{Antibodies}

We used anti-Oct-1 (ab15112, Abcam, Cambridge, UK), Oct-2 (C-20 X, sc-233, Santa Cruz Biotechnology, Santa Cruz, CA), Oct-3/4 (H-134 X, sc-9081, Santa Cruz), mouse Gfilb (D19, sc-8559, Santa Cruz), mouse GATA-1 (N6 X, sc-265, Santa Cruz) antibodies, and rabbit immunoglobulin fraction (normal; X 0903, DAKO, Glostrup, Denmark).

\section{Results}

\section{Gfilb SNP screening}

To gain further insight into Gfilb regulation, we have searched for promoter polymorphisms in 214 patients with hematological abnormalities and 96 controls, blood donors.

We amplified Gfilb promoter, (MN 004188; Fig. 1a) [20] and searched for SNPs through DHPLC followed by sequencing of cases with an abnormal pattern. We found two unknown SNPs (Figs. $1 \mathrm{~b}$ and 2a, b). DHPLC and direct sequencing indicated a similar amount of normal and abnormal alleles (Fig. 2a, b). These were not present in the controls.

Both changes lied in an area of highly conserved sequence through evolution from frog to human (Fig. 2c). One mutation is located at position -8099 (coordinate in relation to the initiation codon), changing the sequence from TGATAA to TGATGA at a GATA-1 binding site previously validated by gel shift and chromatin immuno- precipitation [20]. This change was detected in an acute myeloid leukemia M5a patient at diagnosis and relapse, but it was also found in complete remission bone marrow (data not shown). Additional studies could not be performed, as the patient died after relapse and had no relatives.

The other mutation affected an acute lymphoblastic leukemia patient. It is localized at -8092 (T-C; Fig. 2b, c). Unlike the previous case, in which mouth cell sample was not available, this mutation was detected in mouth cell DNA, indicating that it is congenital. Next, we performed a family study, which concluded that both parents and one sister of the propositus are heterozygous for this base change, and two sisters are homozygous for it $(-8092 \mathrm{C}+/+)$. The family did not report consanguinity, but they are Colombian Caucasians from the same small community in the Andes. Further study of the parents and of both homozygous sisters showed they had normal blood counts (Table 1 of the Electronic Supplementary Material).

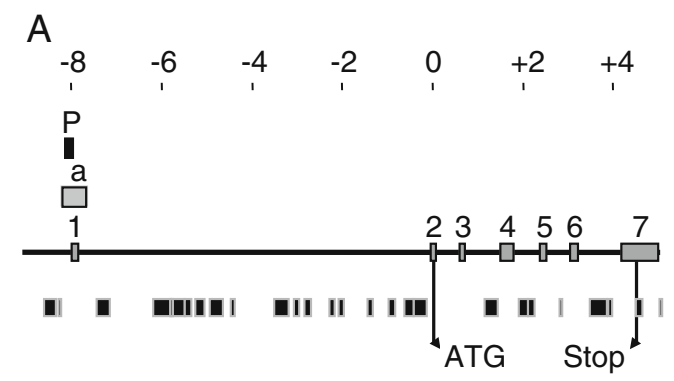

B

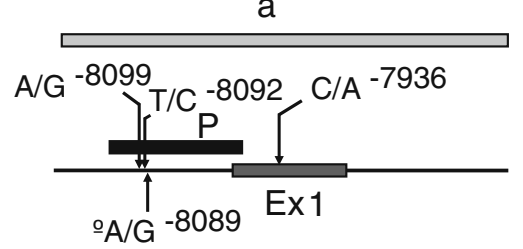

Fig. 1 SNPs are found at the human Gfilb promoter. a Human Gfilb locus. DNA sequence is represented by a black horizontal line. Coordinates are relative to the initiation ATG codon. Gray boxes on the sequence are the exons. Gfilb promoter is shown as a black box over the sequence and the promoter amplicon as a gray box underneath this (a). Black boxes below the sequence are repeats. b Location of Gfilb promoter SNPs. The diagram a. SNPs are indicated with arrows including base changes and the coordinate. ${ }^{\circ}$ SNP included in NCBI database but not found in this study. $P$ promoter, $E x$ exon 
A
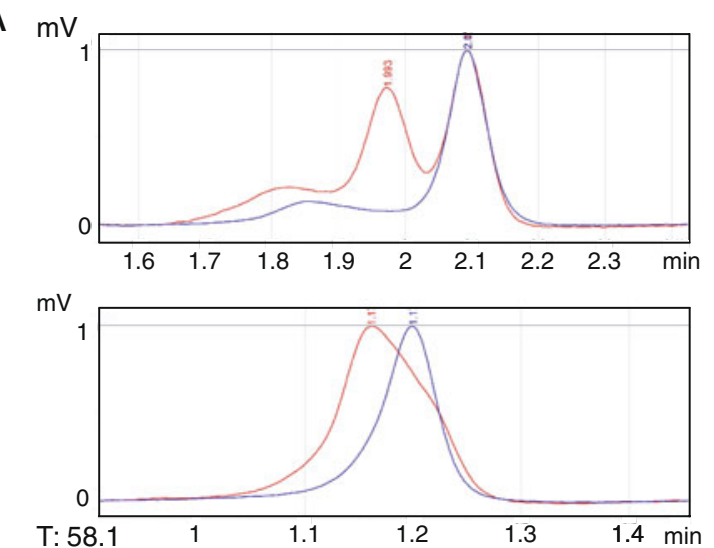

B

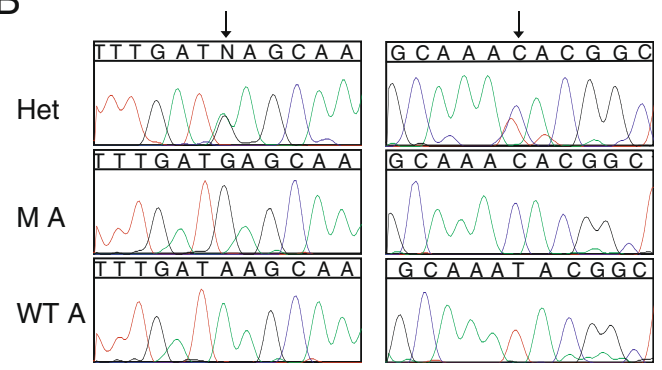

Fig. 2 Promoter mutations localize at conserved sequence motives. a DHPLC tracing of the promoter amplicon from mutant (red) and normal (blue) cases. The top diagrams represent the result obtained after running the samples at $58.1^{\circ} \mathrm{C}$, and the bottom tracings are the consequence of performing the experiment at $60.8^{\circ} \mathrm{C}$. b Sequence analysis of patients with abnormal DHPLC study: on the left-8099 (A$\mathrm{G})$ patient and on the right -8092 (T-C) heterozygous patient. Top direct sequence of PCR products (Het). The middle analyses correspond to the mutant allele after subcloning the PCR products $(M A)$. Bottom wild-

Mutations -8099 (A-G) and -8092 (T-C) affect different protein complexes

To further understand these mutations, we studied protein binding to the normal and mutated sequences by EMSA. An oligonucleotide containing the normal sequence including positions -8099 and -8092 was radioactively labeled and reacted with nuclear proteins from induced MEL and L8057 cells (Figs. 3a, b and Figure 1 of the Electronic Supplementary Material). Two protein/DNA complexes were obtained in both cell lines; they were completely abolished by an excessive amount of unlabeled oligomer. The -8099 (A-G) oligo did not compete completely with any of both complexes, although competed preferentially for the upper complex (I), while -8092 (T-C) oligo inhibited the bottom complex (II) but did not abolish complex I.

Excess amount of unlabeled consensus GATA oligo blocked complex II binding to the probe (Fig. 3a and Figure 1 of the Electronic Supplementary Material) and GATA-1 antibody-inhibited and shifted complexes I and II (Fig. 3b and Figure 1 of the Electronic Supplementary Material). This

C
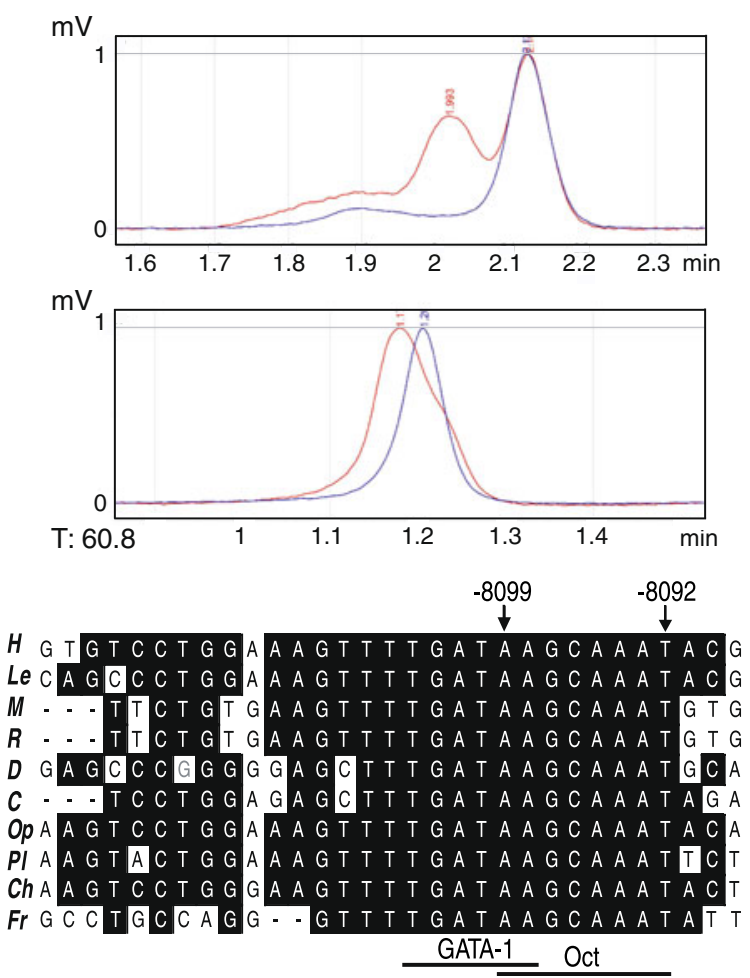

type alleles $(W T A)$. The arrows indicate the position of the mutations. c Sequence alignment of Gfilb promoter including the mutated nucleotides indicated by arrows and coordinates. The alignment was performed using CLUSTALW (MacVector, Accelrys, Cary, NC, USA). $H$ human (Homo sapiens); $M$ mouse (Mus musculus); $R$ rat (Rattus norvegicus); D dog (Canis familiaris); C cow (Bos taurus); $O p$ opossum (Monodelphis domestica); Ch chicken (Gallus gallus). Fr frog (Xenopus tropicalis). GATA-1 and the potential Oct binding sites are underlined

indicates that GATA-1 is present in both complexes, but only complex II directly binds GATA recognition sequence. Mutation -8099 (A-G) decreases this binding, and it also reduces complex I DNA affinity.

Mutation -8092 (T-C) affects a sequence where Oct proteins have been shown to bind that differs for one base to the canonical octamer (ATGCAAAT) [21]. Thus, we used an unlabeled consensus octamer oligomer to compete with normal Gfilb probe; this destroyed complex I (Fig. 3a and Figure 1 of the Electronic Supplementary Material). Antibody against Oct-1 (POU2F1) abolished complex I, suggesting that Oct-1 is part of this complex (Fig. 3b and Figure 1 of the Electronic Supplementary Material).

Gfilb can repress its own transcription, binding to Gfilb promoter [19, 22]. This could be achieved through GATA-1 binding [19]. We incubated the nuclear extracts with an oligonucleotide containing Gfilb binding sequence or Gfilb antibody. These consistently produced an attenuation of the protein/DNA complexes (Fig. 3a, b and Figure 1 of the Electronic Supplementary Material). 
A

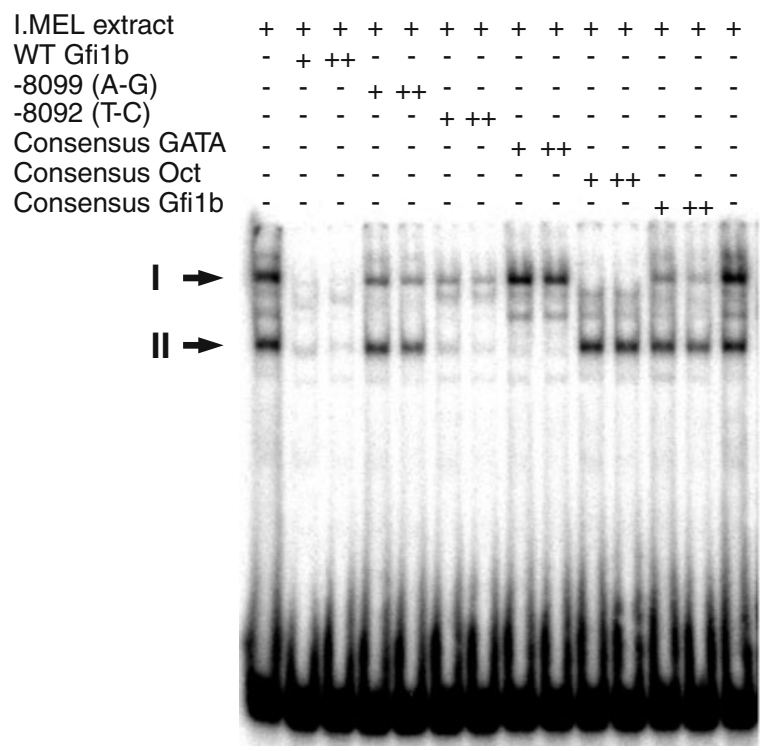

B

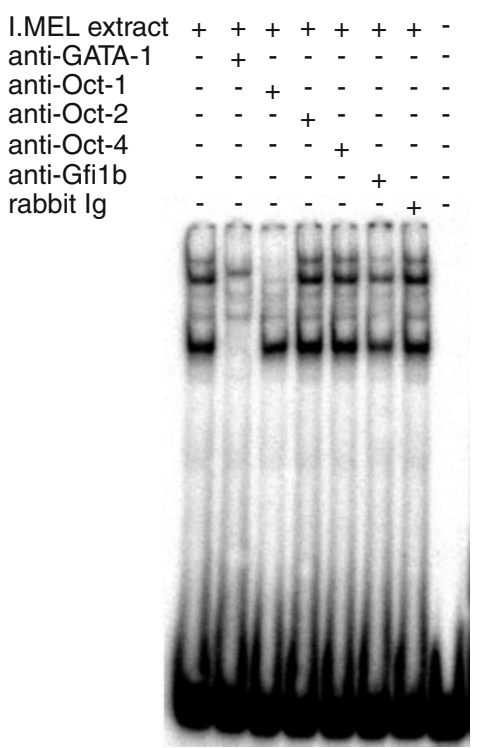

Fig. $3-8099$ (A-G) and -8092 (T-C) mutations mainly affect two different DNA/protein complexes. GATA-1 and Oct-1, respectively, bind to DNA at the sites affected by each of these mutations. Gel shift analysis of the $-8112 /-8081$ region of human Gfilb promoter (coordinates relative to the translation start site). a The ${ }^{32} \mathrm{P}$-labeled double-stranded oligonucleotide corresponding to the $-8112 /-8081$ sequence of Gfilb locus was used for the analysis in the presence or

In conclusion, -8099 (A-G) and -8092 (T-C) mutations mainly affect two different DNA/protein complexes. GATA-1 and Oct-1, respectively, bind to DNA at the sites affected by each of these mutations. Gfilb itself can be part of the complexes under certain circumstances.

Natural occurring mutation at GATA-1 site decreases promoter activity

To study the impact of these changes in vitro, the abnormal and normal alleles were cloned in pGL3-Enhancer Vector (Promega), which encodes firefly luciferase, and then they were transiently transfected to K562 cells. The vector with GATA site mutation consistently decreased luciferase activity to $50 \%$ of the normal (Fig. 4), while the octamer mutation produced some decrease in activity, which cannot be considered significant due to the high interexperimental variability (Fig. 4).

Homozygous Gfilb promoter mutation $-8092(\mathrm{C}+/+)$ increases Gfilb expression in megakaryocytes

To further investigate the role of mutation -8092 (T-C), we studied the impact of this mutation in the erythroid and megakaryocytic lineages. First, we differentiated peripheral blood mononuclear cells from a homozygous individual $(-8092 \mathrm{C}+/+)$ (patient II:1, Fig. 5a) and a normal control absence of unlabeled competitor in $50(+)$ or $100(++)$ fold molar excess with nuclear proteins extracted from induced MEL cells. b Gel shift analysis performed as before; when indicated, antibodies or rabbit immunoglobulins (rabbit $I g$ ) were added. A control with no protein extract is also shown. The arrows indicate the location of complexes I and II

onto erythroid cells in vitro and studied Gfilb and transferring receptor expression in late erythroblasts. Patient and control showed no differences in mRNA levels (Fig. 5b). Next, we assessed Gfilb RNA level in platelets by real-time PCR. We studied three different samples from patient II:1 and one from patient II:2 and compared them with 20 normal individuals (Fig. 5c and Figure 2 of the Electronic Supplementary Material), demonstrating an increase in Gfilb expression more than fourfold in $-8092 \mathrm{C}+/+$ individuals. Control platelet glycoproteins $\mathrm{IIb}$

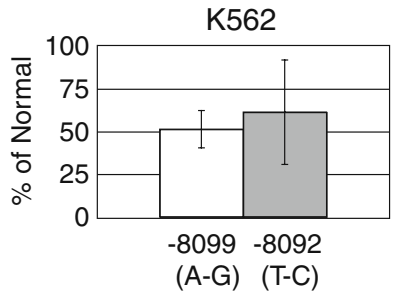

Fig. 4 The -8099 (A-G) mutation causes a reduction in Gfilb expression in vitro. K562 cells were cotransfected with pGL3-Enhancer reporter plasmid containing $-8222 /-7925$ region of wild-type Gfilb, -8099 (A-G) or -8092 (T-C) mutant alleles and pEGFP-C2. Luciferase activity was standardized relative to GFP expression, and data of each mutant allele were plotted relative to wild-type Gfilb promoter result set arbitrarily to 100 . Data from -8099 (A-G) mutation are shown in the white column; the results from -8092 (T-C) mutation are represented in the gray column. Error bars represent \pm standard deviation from the mean of three independent experiments 
A

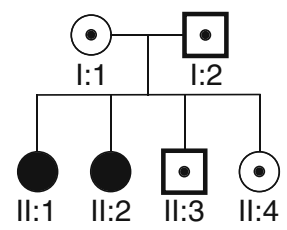

C

Megakaryocytic

p: 0.022

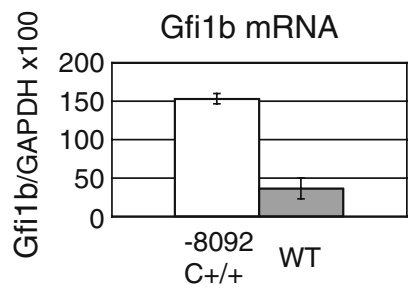

B Erythroid

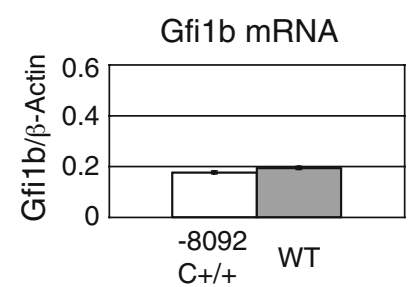

p: 0.648

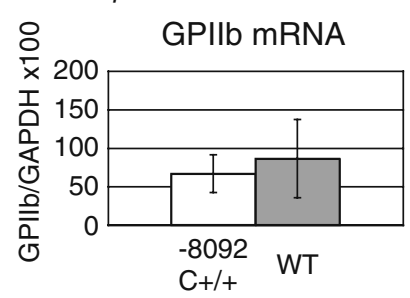

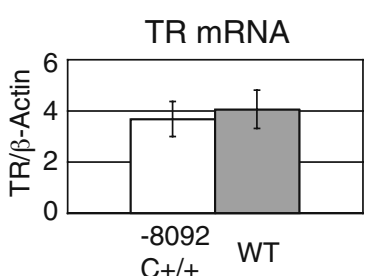

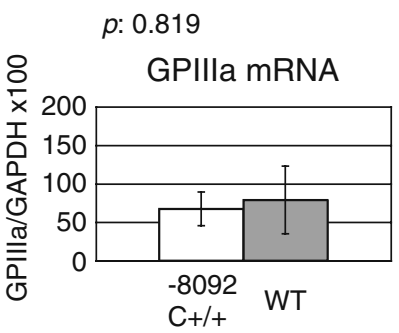

Fig. 5 The $-8092 \mathrm{C}+/+$ mutation associates with increase Gfilb mRNA in platelets but not in erythroid cells. a Pedigree of the family with -8092 (T-C) mutation. Black circles represent $-8092 \mathrm{C}+/+$ homozygous females, circles and squares with black disks represent -8092 (T-C) heterozygous females and males, respectively. b Realtime PCR mRNA analysis of in vitro differentiated erythroid cells. Study on cells after 13 days on second phase culture, when the predominant cells are mainly polychromatophilic and in less proportion ortochromic erythroblasts. Left Gfilb and right transferring receptor $(T R)$; mRNA level was normalized with $\beta$-actin mRNA

and IIIa showed similar RNA levels in $-8092 \mathrm{~T}+/+$ and $\mathrm{C}+/+$ cases (Fig. $5 \mathrm{c}$ and Figure 2 of the Electronic Supplementary Material).

\section{Discussion}

Gfilb promoter mutations indicate that an octamerbinding complex represses Gfilb in the latest stages of megakaryocyte maturation and confirm that a GATA-1containing complex activates the gene. We have recently studied Gfilb regulation through erythropoiesis. As erythroid cells differentiate the general decrease in transcription factor expression, and subsequently of binding to Gfilb promoter and regulatory elements, correlate with progressive reduction in Gfilb expression [23]. Nevertheless, in the megakaryocytic lineage, additional unknown factors must be required to achieve Gfilb repression. This would explain that mutation $-8092(\mathrm{C}+/+)$ increases Gfilb expression in megakaryocytes, but Gfilb level does not change in late erythorid cells, strongly suggesting that Oct-1 is the missing repressor. Accordingly, Oct-1 has intrinsic silencing activity [18]. The octamer-like sequence where the mutation localizes (GATAAGCAAAT to GATAAGCAAAC) partly overlaps the previously described GATA-1 binding site. Likewise, a GATA/Oct expression. Analysis in cells from patient II:1 (-8092 C+/+, white boxes) and a wild-type control (WT, gray boxes) show similar results. The standard deviations correspond to three different studies starting from the cDNA synthesis. c Real-time PCR mRNA study in platelets. Left Gfilb expression in three samples extracted in different days of patient II:1 and one of II:2 (white box) compared to 20 normal controls (gray box), and normalized with GAPDH mRNA level, shows significant differences. Middle panel platelet glycoprotein IIb and right panel glycoprotein IIIa mRNA, studied and plotted as before, showing similar levels of expression in patients and controls

combined site is present at $\gamma$-globin promoter [24]. While this paper was under review, Laurent et al. also described Oct-1 binding to this site together with another position in Gfilb promoter [25]. These authors observed that Oct-1 increases the activity of Gfilb promoter linked to a reporter gene when transfected together with GATA-1 and HGMB2 in HeLa cells. In agreement with our data in erythroid cells, they found a no significant decrease in the promoter activity when the Oct-1 site where -8092 (T-C) localizes was mutated. Nevertheless, Gfilb promoter activity reduced when both Oct-1 sites were destroyed. These in vitro findings may correspond to the assembly of certain protein complexes on Gfilb promoter that can be relevant in vivo in some cell types or stages of differentiation. However, our in vivo data strongly suggest that a repressive complex forms around -8092 in terminally differentiated megakaryocytes.

This is the first report of Gfilb mutations with a functional implication. Considering the evidences that point to the importance of Gfilb hyperexpression in leukemia [6, 7], promoter changes, particularly activating mutations like -8092 (T-C), may be important in blood malignancy. Nevertheless, it is likely that several abnormalities or sequence variations converge to have a pathological impact. Further investigation and follow-up will rule out the involvement of these mutations in hematological disease. 
Acknowledgments The authors would like to thank William G. Wood for the advice, animal samples, and support and Christopher Fisher for technical help, both in Weatherall Institute of Molecular Medicine, Oxford University. We also thank Angel Remacha (Virgen de la Salud Hospital, Toledo, Spain) for patient samples and the Epidemiology Department at Hospital Clinico San Carlos for their help with the statistical analysis.

Authorship and disclosures Contribution: EA was the principal investigator, designed the study, performed the laboratory work, and wrote the paper. AV provided information on the patients. $\mathrm{AH}$ performed some research. The authors reported no potential conflicts of interest.

Funding This work was supported by a grant from the Spanish Ministry of Science (ref. SAF2005-04709/). EA was supported by the Ramón y Cajal Program.

\section{References}

1. Vassen L, Okayama T, Möröy T (2007) Gfílb: green fluorescent protein knock-in mice reveal a dynamic expression pattern of Gfilb during hematopoiesis that is largely complementary to Gfi1. Blood 109:2356-2364

2. Xu W, Kee BL (2007) Growth factor independent 1B (Gfilb) is an E2A target gene that modulates Gata3 in T-cell lymphomas. Blood 109:4406-4414

3. Osawa M, Yamaguchi T, Nakamura Y, Kaneko S, Onodera M, Sawada K, Jegalian A, Wu H, Nakauchi H, Iwama A (2002) Erythroid expansion mediated by the Gfi-1B zinc finger protein: role in normal hematopoiesis. Blood 100:2769-2777

4. Garçon L, Lacout C, Svinartchouk F, Le Couédic JP, Villeval JL, Vainchenker W, Dumenil D (2005) Gfi-1B plays a critical role in terminal differentiation of normal and transformed erythroid progenitor cells. Blood 105:1448-1455

5. Saleque S, Cameron S, Orkin SH (2002) The zinc-finger protooncogene Gfi-1b is essential for development of the erythroid and megakaryocytic lineages. Genes Dev 16:301-306

6. Elmaagacli AH, Koldehoff M, Zakrzewski JL, Steckel NK, Ottinger H, Beelen DW (2007) Growth factor-independent 1B gene (GFI1B) is overexpressed in erythropoietic and megakaryocytic malignancies and increases their proliferation rate. $\mathrm{Br} \mathrm{J}$ Haematol 136:212-219

7. Vassen L, Khandanpour C, Ebeling P, van der Reijden BA, Jansen JH, Mahlmann S, Dührsen U, Möröy T (2009) Growth factor independent $1 \mathrm{~b}$ (Gfilb) and a new splice variant of Gfilb are highly expressed in patients with acute and chronic leukemia. Int $\mathrm{J}$ Hematol 89:422-430

8. Remacha AF, Badell I, Pujol-Moix N, Parra J, Muniz-Diaz E, Ginovart G, Sarda MP, Hernandez A, Moliner E, Torrent M (2002) Hydrops fetalis-associated congenital dyserythropoietic anemia treated with intrauterine transfusions and bone marrow transplantation. Blood 100:356-358

9. Wickramasinghe SN, Wood WG (2005) Advances in the understanding of the congenital dyserythropoietic anaemias. $\mathrm{Br} \mathrm{J}$ Haematol 131:431-446
10. Baxter EJ, Scott LM, Campbell PJ, East C, Fourouclas N, Swanton S, Vassiliou GS, Bench AJ, Boyd EM, Curtin N, Scott MA, Erber WN, Green AR (2005) Acquired mutation of the tyrosine kinase JAK2 in human myeloproliferative disorders. Lancet 365:1054-1061

11. Anguita E, Hughes J, Heyworth C, Blobel GA, Wood WG, Higgs DR (2004) Globin gene activation during haemopoiesis is driven by protein complexes nucleated by GATA-1 and GATA-2. EMBO J 23:2841-2852

12. Ishida Y, Levin J, Baker G, Stenberg PE, Yamada Y, Sasaki H, Inoue $\mathrm{T}$ (1993) Biological and biochemical characteristics of murine megakaryoblastic cell line L8057. Exp Hematol 21:289-298

13. Pope SH, Fibach E, Sun J, Chin K, Rodgers GP (2000) Twophase liquid culture system models normal human adult erythropoiesis at the molecular level. Eur J Haematol 64:292-303

14. De Gobbi M, Anguita E, Hughes J, Sloane-Stanley JA, Sharpe JA, Koch CM, Dunham I, Gibbons RJ, Wood WG, Higgs DR (2007) Tissue-specific histone modification and transcription factor binding in alpha globin gene expression. Blood 110:4503-4510

15. Andrews NC, Faller DV (1991) A rapid micropreparation technique for extraction of DNA-binding proteins from limiting numbers of mammalian cells. Nucleic Acids Res 19:2499

16. Wadman IA, Osada H, Grütz GG, Agulnick AD, Westphal H, Foster A, Rabbitts TH (1997) The LIM-only protein Lmo2 is a bridging molecule assembling an erythroid, DNA-binding complex which includes the TAL1, E47, GATA-1 and Ldb1/NLI proteins. EMBO J 16:3145-3157

17. Gallagher PG, Liem RI, Wong E, Weiss MJ, Bodine DM (2005) GATA-1 and Oct-1 are required for expression of the human alphahemoglobin-stabilizing protein gene. J Biol Chem 280:39016-23

18. Kim MK, Lesoon-Wood LA, Weintraub BD, Chung JH (1996) A soluble transcription factor, Oct-1, is also found in the insoluble nuclear matrix and possesses silencing activity in its alanine-rich domain. Mol Cell Biol 16:4366-4377

19. Huang DY, Kuo YY, Chang ZF (2005) GATA-1 mediates autoregulation of Gfi-1B transcription in K562 cells. Nucleic Acids Res 33:5331-5342

20. Huang DY, Kuo YY, Lai JS, Suzuki Y, Sugano S, Chang ZF (2004) GATA-1 and NF-Y cooperate to mediate erythroid-specific transcription of Gfi-1B gene. Nucleic Acids Res 32:3935-3946

21. Coyle AT, Kinsella BT (2005) Characterization of promoter 3 of the human thromboxane A receptor gene. A functional AP-1 and octamer motif are required for basal promoter activity. FEBS J 272:1036-1053

22. Vassen L, Fiolka K, Mahlmann S, Möröy T (2005) Direct transcriptional repression of the genes encoding the zinc-finger proteins Gfilb and Gfil by Gfilb. Nucleic Acids Res 33:987-998

23. Anguita E, Villegas A, Iborra F, Hernández A. (2009) Gfilb controls its own expression binding to multiple sites. Haematologica 95: $36-46$

24. Liu LR, Du ZW, Zhao HL, Liu XL, Huang XD, Shen J, Ju LM, Fang FD, Zhang JW (2005) T to C substitution at -175 or -173 of the gamma-globin promoter affects GATA-1 and Oct-1 binding in vitro differently but can independently reproduce the hereditary persistence of fetal hemoglobin phenotype in transgenic mice. J Biol Chem 280:7452-7459

25. Laurent B, Randrianarison-Huetz V, Marechal V, Mayeux P, Dusanter-Fourt I, Dumenil D. High mobility group protein HMGB2 regulates human erythroid differentiation through trans-activation of Gfi-1b transcription. Blood. doi:10.1182/blood-2009-06-230094 